\title{
TOYS: AN OPEN NARRATIVE
}

\section{Bronwyn Mohring}

"Toys: An Open Narrative" (2019) is a body of work from my Postgraduate Diploma which explores childhood memories. The pieces created for this project are inspired by toys and the notion of what toys represent. The understanding of an imagined relationship between figurative and allegorical elements is referred to as an open narrative, because the viewer is free to determine a story to infer from the images provided by the artist.

This body of work began with two items as my inspiration: the first, a childhood photograph of myself and my brother on a swing with a significant childhood toy; and the second, the stuffed toy depicted in the photograph, a deer.

"But, what has been the fate of those people in the photographs? Dead or alive, the person in the image is not physically there any more, in that space at that time, which generates an alluring melancholic feeling."'

Through the act of creating objects and images from the past, a fundamental sense of nostalgia emerges - the relevance of it to this tale was revealed as something essential.When we experience nostalgia, our recollections are reconstructed and re-fashioned from fragments of our fallible memories. We do not simply press recall and have instant replay of a particular childhood episode. Rather we reconstruct such episodes piece by piece, often unintentionally mixing and matching recollections as best we can. ${ }^{2}$ Examining our nostalgic memories in a more detached way, we can see the inconsistencies, the hidden realities in them and thus acknowledge memory's essentially creative nature.

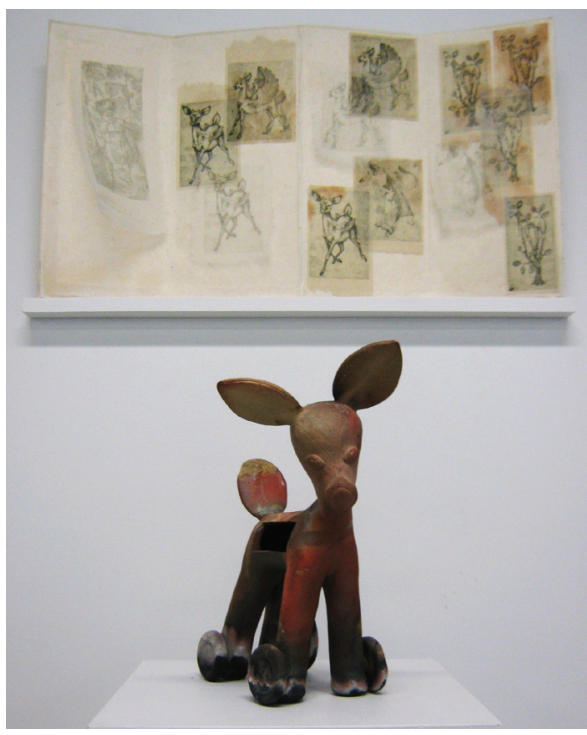

Figure I. Bronwyn Mohring, Flock of Imaginary Deer, 2019 , etchings on transparent papers and fabric; Bronwyn Mohring, Close Memory, 2019. saggar-fired ceramic.

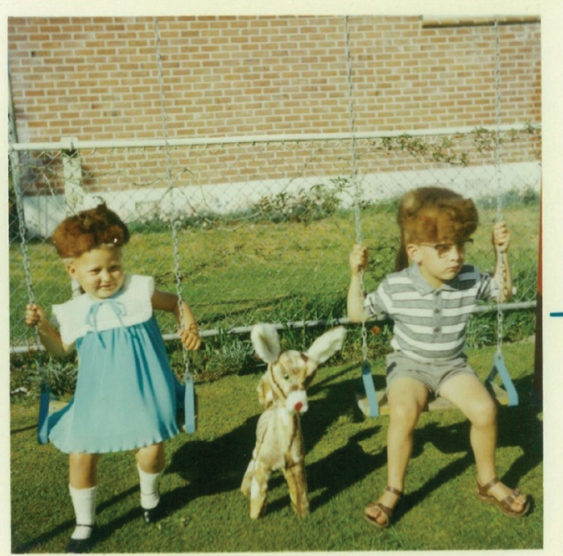

Figure 2. The author and her brother. 
"Photographs, therefore, work as a memento mori, as they confront the death of the person in the image and the unavoidable death of the viewer. In this way, the photographs trigger melancholy in the audience.".3

In this quotation, Domingo Martinez Rosario refers to the power of a photograph to evoke melancholic responses towards the people in the captured image. In my project, the use of photographic imagery is directly exploited to engage the onlooker's empathy for the fate of the subjects. It also serves to disconcert the viewer insofar as the most repeated image in the project is not one of the people in the photograph, but instead is a toy. In this way the destiny of the people depicted in the image, and the inevitability of loss, is referred to obliquely.

In a society where change is the norm and often sudden and dramatic, mundane objects from the past can become treasured souvenirs. When loss becomes imminent, people feel the need to treasure such items as recordings and proof of memory. These familiar items create a sense of security and help preserve our identity in times of loss and change.

In "Toys: An Open Narrative," the reminder of loss through re-inventing images of an old toy and photograph produces a sense of nostalgia. The new sculptural images remain easily identifiable as inspired by the original, but they are also significantly changed. Through shared history, imitation of image and shared handmade creation, the sculptures become new custodians of the memories of the old toy, retaining links to others who have touched, gifted and shared its history. While the change in medium from the original soft textile to clay and glass is achieved through the use of techniques to embrace the sense of softness, the reality of replacing soft fabric with hard and fragile clay and glass creates a frisson which addresses the fragility and unreliability of memory.

This presentation acknowledges childhood nostalgia as a very subjective experience, full of symbols with diverse meanings - powerful for one and mysterious to another. This installation of ceramic, glass and printed images presents personal nostalgic memories in the form of familiar images and symbols. This directness is intended to invoke empathy from the viewer and encourage engagement with the project. However, other symbols and meanings come straight from the subconscious, bound by feeling alone, and remain indefinable in words, even to the maker.

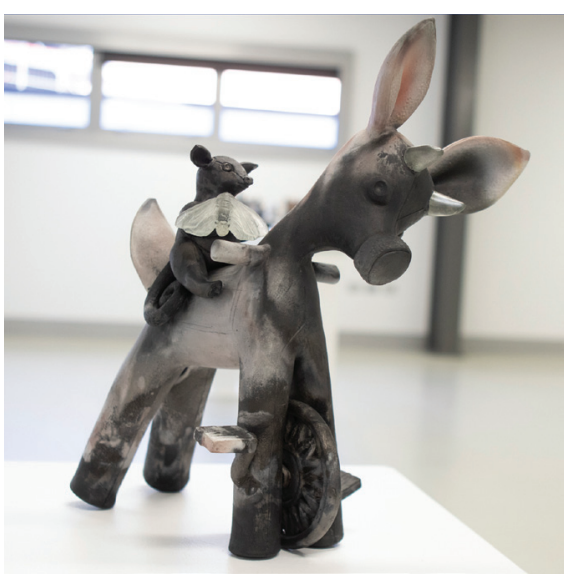

Figure 3. Bronwyn Mohring, Memory Becomes Fable, 2019, saggar-fired ceramic and cast glass. Photograph: Jodie Gibson.

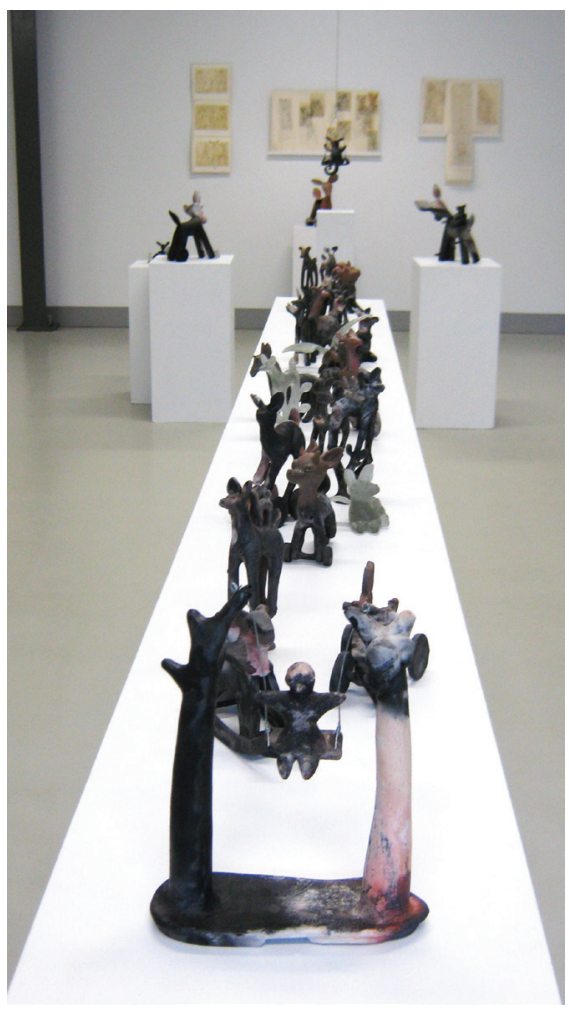

Figure 4. Bronwyn Mohring, "Toys: An Open Narrative." Installation view. SITE Exhibition, Dunedin School of Art, 2019. 
Studying my own history, and the significant place of toys in it, led me to examine toys of other cultures, times and histories. When comparing ancient toys with their modern counterparts, the surprising commonalities of form and function provide a touching awareness of a shared human experience, still palpable despite the difference of time periods. Children of all nationalities and times form special attachments to items, either specifically designed for play or gathered by the child from their surroundings and given the identity of a toy through their use in play. This commonality of childhood experience means that often toys dominate our perception of childhood itself.

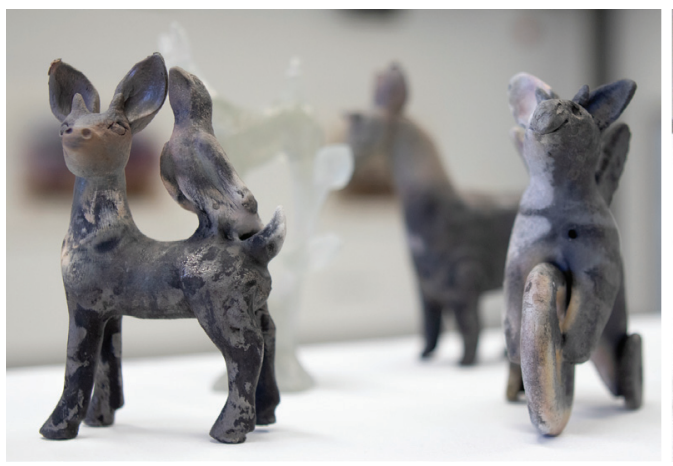

Figure 5. Bronwyn Mohring, selection from Army of Imaginary Deer, 2019, saggar-fired ceramic. Photograph: Jodie Gibson.

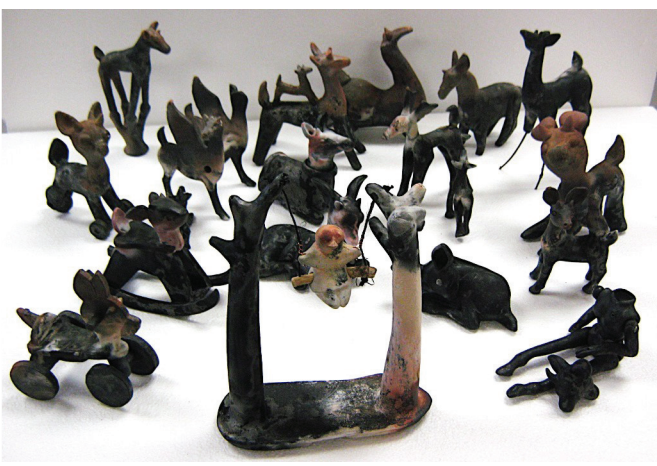

Figure 6. Bronwyn Mohring, selection from Army of Imaginary Deer, 2019 , saggar-fired ceramic.

One of the ceramic groups in this project expresses how the child in the photograph perceived the toy deer. It is a collection of ceramic 'sketches' containing the elements of all the imaginary necessities that the toy provides the child. It consists of a group of deer figures which portrays the motif of the deer, the doe, in myth, history and art. Some of the figures are direct references to ancient Hellenistic toys in the form of wheeled animals, which here stand beside modern interpretations of the wheeled toy animal. Others are interpretations of ancient stags in bronzes re-imagined in ceramic form as does. Some of the images are inspired by prehistoric statues displayed in museums alongside copies found in their associated tourist shops. Others again are inspired by contemporary children's toys and modern sculpture.

Another essential element in the original childhood photograph is the swing. A swing is almost a timeless toy, an ancient and modern time traveller; and so the child on the swing becomes a time traveller also. It's the swing that frames the photograph and unites the characters. The clay child/bird on a swing depicted in this project is a direct reference to the child in the photograph. It is such a universal image that the swinging child becomes any and every child.

Whether inspired by comforting toys or wild animals or ornament or totemic figures, these pieces are united by the saggar firing technique used and the child figure who imagines them. The swinging child figure is timeless, and so her army of deer is also timeless.

In his article "Design History and the History of Toys," Anthony Burton claims that the history of toys only became the focus of serious study from 1900, when the child study movement began to develop a significant academic interest in child development. ${ }^{4}$ However, the importance of play and games as a social and cultural phenomenon was recorded in theoretical writings in the West dating back to the eighteenth century. Philosophers Immanuel Kant (1776-77), and Friedrich Schiller (1759-1805) both wrote on the importance of games. According to French anthropologist and philosopher Charles Brougere (1955-), "Toys deserve to be studied for themselves, since they are important objects for what they reveal of a culture."' 
Both the givers and receivers of toys acknowledge their significance. They often constitute a form of non-verbal communication between adult giver and child receiver. A toy is any material object designated as a plaything. Although toys reflect the society which produces them, to the child they are an intimate and personal companion in their personal private world. The importance of the toy to the child does not necessarily reflect what the toy is intended (by its maker) to represent, but is instead what the child perceives it needs to be in the present moment. The toy's identity is incorporeal, although the object itself is material.

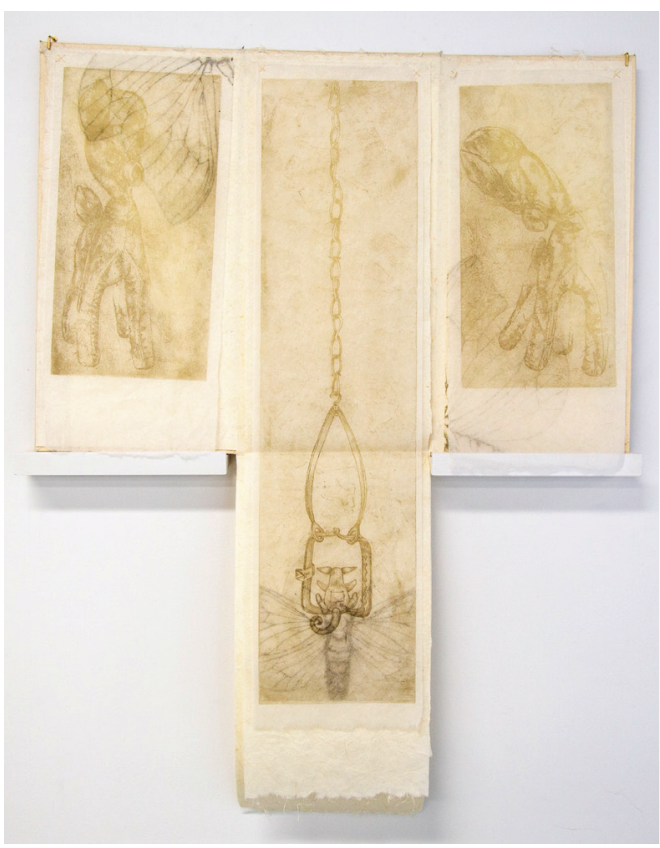

Figure 7. Bronwyn Mohring,

The Parable of the Possum and the Swing, 2019, etchings on transparent papers in book form.

Photograph: Jodie Gibson.

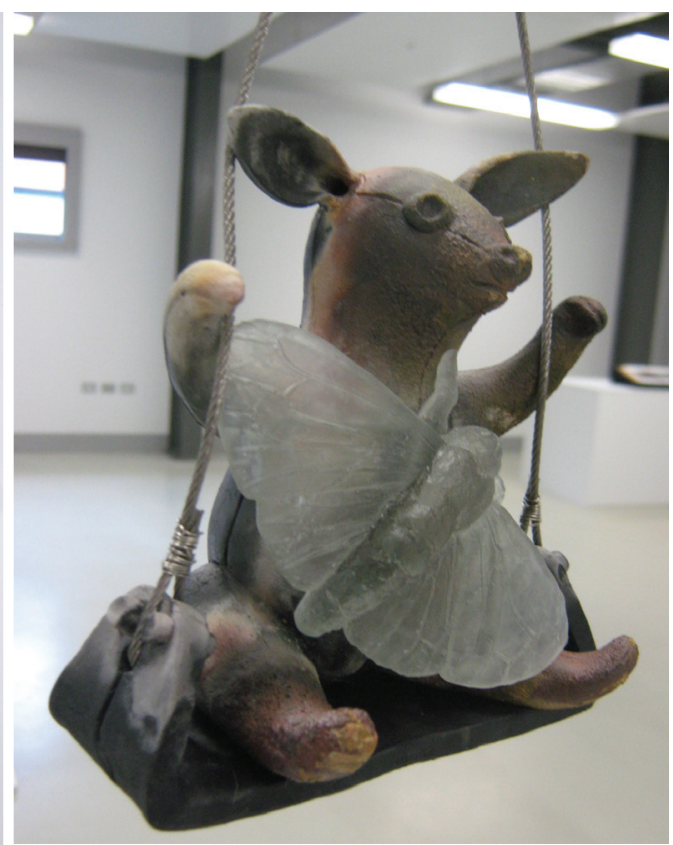

Figure 8. Bronwyn Mohring, Before the Fall, 2019 , saggar-fired ceramic and cast glass.

The Collins Dictionary defines "narrative" as a noun meaning an account of events, and as an adjective referring to the telling of a story. ${ }^{6}$ Narrative can be seen as synonymous with tale, yarn, legend and chronicle. A narrative or story may be an account of a series of related events, but "a story" is also commonly used to mean gossip, rumour and even lies. In "Toys: An Open Narrative," I am relating to the process of story-telling where a series of events is symbolised with identifiable characters, but their relationship is evolving and open to interpretation. Because the relationship between the characters is unverifiable and reliant on interpretation and fallible memory, their story remains open, unable to distance itself from rumour and invention. Instead of struggling to decipher truths with no one to verify them, this project delights in the ability of memory to create new narratives and provide stories in which the inner psyche can delight.

In "Toys: An Open Narrative," the three accordion book forms contain etchings on transparent paper, which is also utilised to embrace the narrative qualities of the print work. In the book of etchings, Before the Fall, the swinging possum toy seems unaware of the precarious nature of his trap-like swing; the old and young toy deer both look on, aware that playing too intimately with the ghost moth is perilous. 
Finding the photograph and the toy that sparked this project after many years was like accessing something I had accepted as faded and ghostly, and discovering that it was suddenly visible, tactile, and had become newly relevant. Vague memories whose validity I had begun to question suddenly become vivid, holding far more truth than I had faith to believe. The intimacy of the moment captured by the camera became not just a memory, but something recorded and able to be shared and held up as proof of truth. However, these tangible 'things', (the photo, the toy) still contain qualities of the ephemeral and are still deeply fragile. The toy is wilted, the plush coating nearly gone, it is almost falling. The photograph only holds images of the past and has its own limited lifespan.

The toy deer has reappeared, enabling me to better recall my own past, but she also carries memories of my family. In the photograph, my brother swings with me, but it also evokes memories of my sister who didn't make it, and my great auntie who gifted me the deer.Their reappearance has led me to re-examine the photograph - not just with a grieving nostalgia, but with an adult's knowledge of the characters involved in this still life. The reappearance of the deer toy has meant the return of my sister, brother and my great aunt back in my life. That is a lot for a stuffed toy to carry on its back.

Having the photograph and toy returned to me is like cuddling a ghost and greeting all its ephemeral 'hangers on; which emerge from the shadows the more I become reacquainted with them. The larger ceramic forms are a representation of how I perceive the toy and all the characters in the story as an adult.

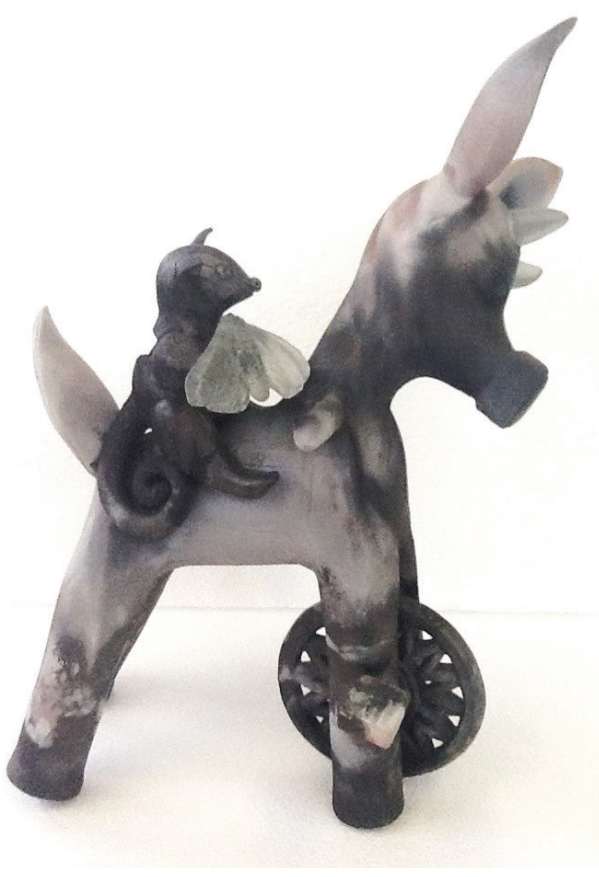

Figure 9. Bronwyn Mohring, Lucid Dream, 2019, saggar-fired ceramic and cast glass.

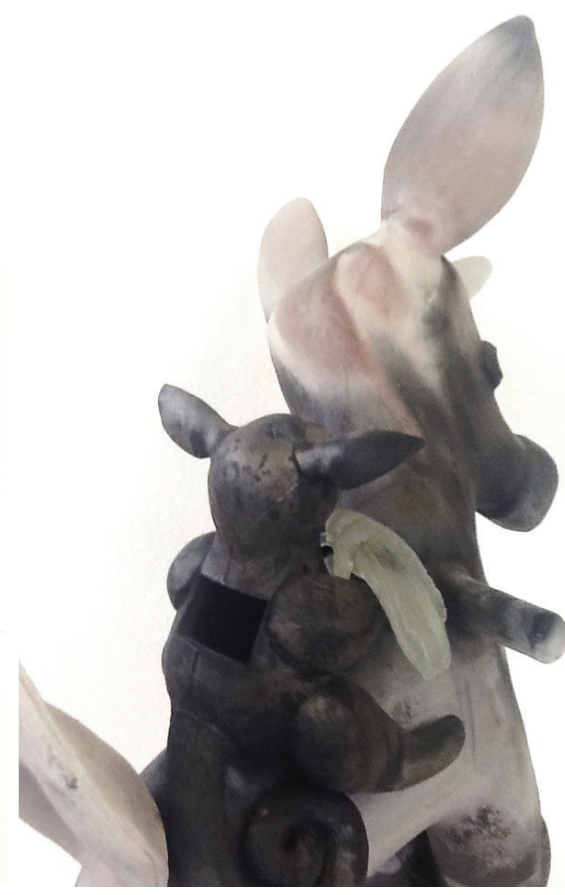

Figure 10. Bronwyn Mohring, Lucid Dream, 2019,

saggar-fired ceramic and cast glass. 


\section{CONCLUSION}

In "Toys: An Open Narrative," I used both print and sculpture to examine issues related to memory. The project pays particular attention to the relevance and role of toys in coming to terms with our limited understanding of our surroundings as a child, and the recognition of emergent issues as these memories are re-examined as an adult. The sculpture combines clay with glass - the transparency to explore the fragility of memory and of life, and clay to embrace our more tangible selves. Both mediums are fragile, and my use of multimedia embraces the idea that memory is not captured and frozen, but layered and living. The print elements of this project explore animal and toy forms with personal, human allusions, and present a dream-world narrative in a simple book form. The project's storytelling qualities were also important in making decisions about the compositional aspects of their presentation.

The body of work I have so far created for this project takes the form of incomplete narratives inspired by toys. Toys are perceived as a safe and sympathetic conduit for sharing nostalgia and memories. Toys, gifted by hand for hands to use, are invitations to participate in the world of the toy. They create a fissure in our mundane world and invite daydream and speculation. They are by nature both ancient and modern, and also constitute an open narrative. The tension between the familiar and the elusive presented in the narrative symbology of this project is explored in the use of both common and hidden imagery. "Toys: an Open Narrative" aims to celebrate the place of toys in childhood, as well as the place they have in the nostalgic memories of adults.

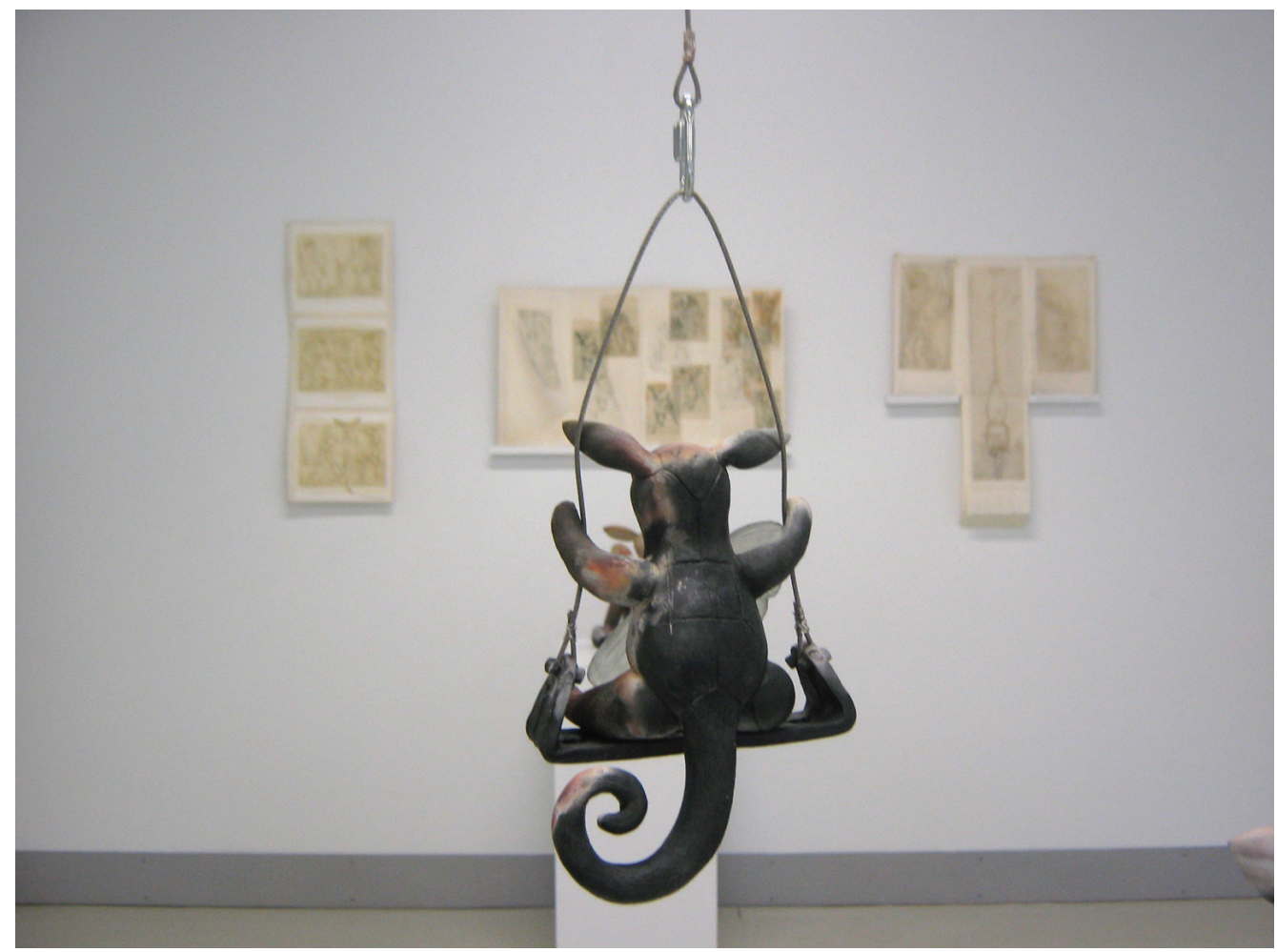

Figure II. Bronwyn Mohring, Before the Fall, 2019 , saggar-fired ceramic and cast glass (with etchings in book form). 
Bronwyn Mohring graduated from Christchurch Polytechnic with a Diploma in Craft Design in 1994 and with a Diploma of Ceramic Design from Otago Polytechnic in 1995. She has worked in the sculptural pottery field ever since. In 2019 Bronwyn return to the Dunedin School of Art to refresh her skills and face new challenges while working towards her Master of Fine Arts.

I Domingo Martinez Rosario, "Representation of Nostalgia and Melancholy in Contemporary Artworks: Tacita Dean and Louise Bourgeois," unpub. PhD diss. (Nottingham Trent University, Rovinj, Croatia, 20 I5).

2 Hal McDonald, "The Art of Nostalgia: Nostalgia as an Aesthetic Form of Memory," Psychology Today, 10 October 20 I7, https:// www.psychologytoday.com/us/blog/time-travelling-apollo/2017।0/the-art-nostalgia.

3 Martinez Rosario, "Representation of Nostalgia."

4 Anthony Burton, "Design History and the History of Toys: Defining a Discipline for the Bethnal Green Museum of Childhood," Journal of Design History, I0:I (1997), I-21, https://academic.oup.com/jdh/article/ I0/1/1/499 I 3 I.

5 Gilles Brougère, Binquedo e Cultura [Games and Culture] (São Paulo: Cortez, 20 I0), 44.

6 Collins Concise Dictionary and Thesaurus (Glasgow: HarperCollins, 1995), 633. 